\title{
An Adaptive Robust Control Method for Trajectory Tracking of a 5 DOF RLED Robot Manipulator
}

\author{
Mohammad Eghtesad ${ }^{1}$, Mohsen Mohseni ${ }^{1}$, and Amir Lotfavar ${ }^{2}$ \\ ${ }^{I}$ (School of Mechanical Engineering, Shiraz University, Shiraz, Iran) \\ ${ }_{2}^{2}$ (Department of Mechanical and Aerospace Engineering, Shiraz University of technology, Shiraz, Iran \\ and Visiting Professor at Ryerson University)
}

\begin{abstract}
In this paper, combined dynamic equations of motion of a rigid-link non-redundant $n$-DOF robot manipulator consisting of mechanical links, all with revolute joints, and electrical actuators are considered and application of an adaptive robust control algorithm is proposed for trajectory tracking of the robot manipulator. Analysis of stability of the suggested scheme is presented in details. The simplicity of the control law and low computational load are two main advantages of the proposed method. Simulation results of applying this technique on a 5 DOF RLED robot illustrate the merits of the scheme and show that the algorithm achieves the specified tracking precision without any a priori information on disturbances or system parameters uncertainty.

Keywords: Adaptive-Robust control, Actuator dynamics, Robot control, Trajectory tracking, RLED robot manipulator, Stability.
\end{abstract}

\section{Introduction}

The In robotic systems, different types of actuators are utilized to drive the links, wheels, legs, etc. Usually, electrical actuators are utilized. Electrically driven robot manipulators can be controlled in two different levels: torque level and voltage level. When one uses a torque-level controller, actuator dynamics is disregarded which implies a simplified (typically linear) relation between torque and voltage vectors is assumed.

Robot's actuator dynamics constitutes a significant part of the complete robot dynamics and becomes extremely important during situations in which motor currents are rapidly changing. Recently, actuator dynamics has been explicitly included in robot manipulator control schemes, [1-8]. It has been shown that this inclusion is necessary in order to have high controller efficiency, [9]. However, the inclusion increases the order of equations of system dynamics and complicates the controller structure and its stability analysis, [10].

Most of model based control schemes proposed for rigid-link electrically-driven (RLED) robot manipulators are different versions of adaptive and robust controllers. One of the attractive features of the adaptive controllers is that the control implementation does not require a priori knowledge of unknown constant system parameters. In a robotic system some of the parameters such as payload mass or friction coefficients are difficult to compute or measure, therefore, adaptive controllers represent an important step toward highspeed/precision robotic applications, [11]. Several direct adaptive algorithms have been presented for trajectory tracking of RLED robots in the literature. In some of them full knowledge of the actuator parameters is required and only mechanical parameters are assumed to be uncertain, [12], and in some of them, both mechanical and electrical parameters are considered with uncertainty [2, 5, 6, 13-15]. Yuan followed the latter case, [6]. In his work, the torque vector is no longer an input vector; instead, it is an output signal controlled by the voltage commands such that it converges to a desired (calculated) torque vector, $\tau_{d}$. In this approach, like the approach presented in [12], acceleration feedback vector is needed, but this signal is seriously corrupted with noise. This limitation can be removed by estimating acceleration instead of measuring it, [6]. Also, required computational load is very high, because of the need for $\dot{\tau}_{d}$ to be calculated. In the approach proposed by Stepanenko and $\mathrm{Su}$, the design procedure is a two-step process, [14]. First, the current vector is regarded as a control variable for mechanical subsystem and an embedded control input for the desired current vector, Id, is designed so that the tracking goal may be achieved. Second, the voltage commands are designed such that I tracks $\mathrm{I}_{\mathrm{d}}$. This procedure can be viewed as a backstepping approach, [3], and requires the measurements of motor currents, joint coordinates and velocities vectors. In a series of works by Dawson et al., including [2, 13-14], they presented an adaptive method together with a pseudo-velocity filter to eliminate the need to measure the joint velocities. In this case, only joint coordinates and motor currents should be measured. Two disadvantages of the adaptive controllers are: 1) large amount of on-line calculation is required (especially for higher DOF robots), and 2) lack of robustness to additive bounded disturbances, [11, 16], and these are true for those works.

Two of the attractive features of the robust controllers are 1) on-line computation is kept to minimum and 2) their inherent robustness to additive bounded disturbances, [11, 14-16], used robust controllers and bounds on the parameters uncertainty and not adaptation mechanisms to estimate them. One of the 
disadvantages of the robust control approaches is that these controllers require a priori known bounds on the uncertainty. In general, calculation of the bounds on the uncertainty can be quite a tedious process since this calculation involves finding the maximum values for the inertia and friction related constants for each link of the robot manipulator. Another disadvantage of the robust control approaches is that even in the absence of additive bounded disturbances, asymptotic stability of the tracking error cannot be guaranteed. In general, it would be desirable to obtain at least a "theoretical" asymptotic stability result for the tracking error, $[11,16]$.

In this paper, an adaptive robust control method is developed for tracking control of RLED robot manipulators. The adaptive robust controller can be thought of as combining the best qualities of the adaptive controllers and the robust controllers. This control approach has the advantages of: 1) reduced on-line calculations (compared to the adaptive control methods), 2) robustness to additive bounded disturbances, 3) no need to a priori knowledge of system uncertainty, 4) simplicity of the control commands and 5) asymptotic tracking error performance. The method presented in [14], is an adaptive robust controller, but its on-line calculation for estimating the parameters is not reduced in compare to its adaptive counterpart, [14].

This paper is organized in the following sections. In section 2, dynamic equations of motion of a general RLED robot manipulator including its mechanical links (second order vector ODE) and its electrical actuators (first order vector ODE) are presented and then combined to obtain a set of third order ODE's. Also, the properties of robot's dynamics are reviewed. In section 3, the adaptive-robust algorithm is presented for mechanical arm dynamics and then its extension for a general RLED robot is proposed. Details of stability analysis of the suggested method are presented in section 4.

A 5 DOF RLED robot is considered as an example to apply the adaptive robust algorithm. Some of the robot's specifications are given in section 5. Results of simulation of trajectory tracking of the 5 DOF robot for the given desired trajectory using the proposed scheme for two cases: 1) without any disturbances and 2) with sinusoidal vector of input voltage disturbances are presented in section 6 . The paper concludes in section 7.

\section{Robot Arm and Actuator Dynamic Equations}

The dynamic model of a general rigid $n$-link robot is a second order nonlinear differential vector equation $[11,16-17]$ :

$$
M(q) \cdot \ddot{q}+C(q, \dot{q}) \cdot \dot{q}+g(q)=\tau
$$

where $q \in R^{n}$ is the vector of joint space generalized coordinates; $\tau \in R^{n}$ is the vector of generalized torques; $M(q), C(q, \dot{q}) \in R^{n \times n}$ and $g(q) \in R^{n}$ are the inertia matrix, matrix of centrifugal and Coriolis effects and vector of gravity terms, respectively.

$M(q)$ is an $\mathrm{n}^{\times} \mathrm{n}$ symmetric positive definite matrix, bounded below and above, i.e. $\exists \alpha \geq \beta>0$, such that $\beta \leq\|M(q)\| \leq \alpha, \forall q \in R^{n}$, (property 1). In addition, $C(q, \dot{q})$ and $g(q)$ are above bounded, i.e. $\|C\| \leq c_{b}(q) \cdot\|\dot{q}\|^{2}$ and $\|g(q)\| \leq g_{b}(q)$, where $c_{b}(q)$ and $g_{b}(q)$ are known scalar functions that for a manipulator with only revolute joints are constant but for a manipulator with some prismatic joints may depend on $q$, (properties 2, 3, respectively). The matrix $\dot{M}(q)-2 . C(q, \dot{q})$ is skew-symmetric by a proper definition of $C(q, \dot{q})$, namely: $x^{T} \cdot[\dot{M}(q)-2 \cdot C(q, \dot{q})] \cdot x=0 \quad \forall x \in R^{n}$, (property 4). The dynamic parameter linearability enables one to write the dynamic equations in the linear-in-parameters (LIPs) form:

$$
M(q) \cdot \ddot{q}+C(q, \dot{q}) \cdot \dot{q}+g(q)=Y(q, \dot{q}, \ddot{q}) \cdot \varphi
$$

where $Y(q, \dot{q}, \ddot{q}) \in R^{n \times p}$ is known as regressor matrix and $\varphi \in R^{p}$ is the vector of robot base dynamic parameters, (property 5) .

With DC motors as system actuators, actuator dynamics is a first order differential equation:

$$
L . \dot{I}+R \cdot I+K_{e} \cdot A \cdot \dot{q}=v
$$

where $L, R, K_{e}$ and $A$ are electrical inductance, electrical resistance, back emf coefficient and gear ratio diagonal matrices, respectively. $I$ and $v$ are armature current and motor input voltage vectors, respectively. Relation between the vector of joint generalized torques and the vector of armature currents is described by:

$$
\tau=A . K_{T} . I
$$

where $K_{T}$ is positive definite constant diagonal matrix of actuator torque coefficients. Dimensions of these matrices and vectors are considered as appropriate.

One may omit $\tau$ by combining equations (1) and (4). Taking derivative of the consequent equation and eliminating $i$ by combining the derivative relation and equation (3) will lead to the following third-order dynamics of the arm and actuators:

$$
H(q) \cdot \ddot{q}+B(q, \dot{q}) \cdot \ddot{q}+C^{\prime}(q, \dot{q}, \ddot{q}) \cdot \dot{q}+D(q, \dot{q}, \ddot{q})=v
$$


Where

$$
\begin{aligned}
& \begin{aligned}
H(q)= & L K_{T}^{-1} A^{-1} \cdot M(q)=L^{\prime} \cdot M(q) \\
B(q, \dot{q})= & L K_{T}^{-1} A^{-1} \cdot(\dot{M}(q, \dot{q})+C(q, \dot{q}))+R K_{T}^{-1} A^{-1} \cdot M(q) \\
& =L^{\prime} \cdot(\dot{M}(q, \dot{q})+C(q, \dot{q}))+R^{\prime} \cdot M(q)
\end{aligned} \\
& \begin{aligned}
C^{\prime}(q, \dot{q}, \ddot{q}) & =L K_{T}^{-1} A^{-1} \cdot \dot{C}(q, \dot{q}, \ddot{q})+R K_{T}^{-1} A^{-1} \cdot C(q, \dot{q})+K_{e} \\
& =L^{\prime} \cdot \dot{C}(q, \dot{q}, \ddot{q})+R^{\prime} \cdot C(q, \dot{q})+K_{e}
\end{aligned} \\
& \begin{aligned}
D(q, \dot{q}, \ddot{q}) & =L K_{T}^{-1} A^{-1} \cdot \dot{g}(q, \dot{q})+R K_{T}^{-1} A^{-1} \cdot g(q) \\
& =L^{\prime} \cdot \dot{g}(q, \dot{q})+R^{\prime} \cdot g(q)
\end{aligned}
\end{aligned}
$$

Since $M(q), K_{T}, L$ and $A$ are symmetric positive definite matrices, $L^{\prime}, R^{\prime}$ and $H(q)$ will be symmetric positive definite matrices, too. Also, the gear ratios of the motors may be chosen in such a way that the elements of the diagonal matrix $L^{\prime}$ are equal, then $L^{\prime}=l^{\prime} . I_{n \times n}$, where $I_{n \times n}$ is the $\mathrm{n} \times \mathrm{n}$ identity matrix.

\section{Adaptive Robust Control of RLED Robots}

In order to present an adaptive robust control method for a (robotic) system, one may begin with an adaptive algorithm and then try to make it robust with respect to some uncertainty and disturbances. Also, one can start with a robust controller by using some bounds on the uncertainty and disturbances and then use an adaptive mechanism to estimate the bounds (not the uncertainty or disturbances).

In this paper, using the latter approach, an adaptive robust control method that decreases much of computational complexity and has a simple controller vector of voltage commands is proposed for trajectory tracking of RLED robots. Let's begin with a robust approach for mechanical arm dynamics, Eqn. 2, and then put forward an adaptive law for bounds estimation, and at the end, extend the work to combined robot dynamics, Eqn. 5.

Robust passivity based control law for the system of Eqn. 2, has been suggested as follows, [16]:

$\tau=\hat{M} \ddot{\zeta}+\hat{C} \dot{\zeta}+\hat{g}-K_{D} \sigma+u_{0}$

where $\hat{M}, \hat{C}, \hat{g}$ have the same forms as $M, C$ and $g$, respectively, but with estimated parameters, $K_{D}$ is a constant positive definite matrix, and

$$
\begin{aligned}
& \dot{\varsigma}=\dot{q}_{d}-\Lambda \tilde{q} \\
& \sigma=\dot{q}-\dot{\varsigma}=\dot{\tilde{q}}+\Lambda \tilde{q}
\end{aligned}
$$

where $\Lambda$ is a constant positive definite matrix, $q_{d}$ is the desired trajectory and $\tilde{q}=q-q_{d}$ is vector of position tracking error.

Eqns. (2) and (7) give:

$\hat{M} \dot{\sigma}+\hat{C} \sigma+K_{D} \sigma=\tilde{w}+u_{0}$

where (see property 5 )

$\tilde{w}=Y^{\prime} \tilde{\varphi}=(\hat{M}-M) \ddot{\zeta}+(\hat{C}-C) \dot{\zeta}+(\hat{g}-g)$

and

$\tilde{\varphi}=\hat{\varphi}-\varphi$

in which $\hat{\varphi}$ and $\varphi$ are the vectors of estimated and exact base dynamic parameters of the system, respectively. It has been shown that the Lyapunov function, $V=1 / 2 \sigma^{T} M \sigma$, has negative semi-definite time derivative if $u_{0}$ is chosen as, [16]:

$u_{0}=-\left(\rho^{2} / \varepsilon\right) \sigma$

Also, $u_{0}$ can be considered as, [11]:

$u_{0}=-\left(\rho^{2} /(\varepsilon+\rho\|\sigma\|)\right) \sigma$

where $\varepsilon$ is a vector of positive valued functions and

$\|\tilde{w}\| \leq \rho$

and from properties 1,2 and 3, [16]:

$\rho=\alpha_{0}+\alpha_{1}\|\tilde{q}\|+\alpha_{2}\|\dot{\tilde{q}}\|+\alpha_{3}\|\dot{\tilde{q}}\|^{2}$

or, [11]:

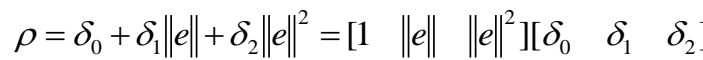


where $\mathrm{e}=\left[\begin{array}{cc}\tilde{q} & \dot{\tilde{q}}\end{array}\right]$, and (when only revolute joints are used) the constant bounds $\alpha_{i}$ 's and $\delta_{i}$ 's depend on $K_{D}, \Lambda$, desired trajectory, $\hat{M}, \hat{C}$ and $\hat{g}$ and can be a priori calculated in a complicated and lengthy process, [16].

To find an adaptive mechanism for this system, first there should be recognized some uncertain parameters to be estimated on-line. These parameters could be either $\hat{\varphi}$ or $\hat{\theta}$. For an adaptive-robust controller, to estimate a fewer number of unknowns, one may choose the vector of uncertain bounds, $\hat{\theta}$. But, there is a problem for choosing $\hat{\theta}$ and that is $\hat{\varphi}$ is not estimated anymore and, therefore, its terms cannot be used in equation (7). To deal with this problem and to make the controller (torque) commands simpler, one may formulate the following changes:

$$
\begin{aligned}
& \tau=-K_{D} \sigma+u_{0} \\
& M \dot{\sigma}+C \sigma+K_{D} \sigma=-M \ddot{\zeta}-C \dot{\zeta}-g+u_{0} \\
& =-Y^{\prime} \varphi+u_{0}=w^{\prime}+u_{0} \\
& \left\|w^{\prime}\right\| \leq \rho
\end{aligned}
$$

Note that the equations (16) and (17) need not to be changed, although the constant bounds do not have their previous values. These parameters can be updated by the following adaptation mechanism:

$$
\dot{\hat{\theta}}=\dot{\tilde{\theta}}=-\gamma S^{T}\|\sigma\|
$$

where $\gamma$ is a positive definite matrix, $\tilde{\theta}=\hat{\theta}-\theta$, and $\hat{\rho}$ and $\tilde{\rho}=\hat{\rho}-\rho$ can be defined as:

$$
\hat{\rho}=S \hat{\theta} \quad, \quad \tilde{\rho}=S \tilde{\theta}
$$

The following Lyapunov function can be used for stability analysis of the system:

$$
V=1 / 2 \sigma^{T} M \sigma+1 / 2 \tilde{\theta}^{T} \gamma^{-1} \tilde{\theta}+K_{\varepsilon}^{-1} \varepsilon
$$

where $K_{\varepsilon}$ is a positive definite matrix. Details of taking time derivative of the Lyapunov function are given in the appendix. After some substitutions and algebraic manipulation, the Lyapunov function time derivative will be obtained as:

$$
\dot{V} \leq-\sigma^{T} K_{D} \sigma
$$

The eigenvalues of $K_{D}$ should be chosen by a trial and error procedure so that the closed-loop system is fast enough to work for the robotic system and, on the other hand, it is computationally stable.

This approach can be extended to combined robot dynamics, Eqn (5). By modifying definitions of $\varsigma$ and $\sigma$ as:

$$
\begin{aligned}
& \ddot{\zeta}=\ddot{q}_{d}-\Lambda_{1} \dot{\tilde{q}}-\Lambda_{2} \tilde{q} \\
& \sigma=\ddot{q}-\ddot{\zeta}=\ddot{\tilde{q}}+\Lambda_{1} \dot{\tilde{q}}+\Lambda_{2} \tilde{q}
\end{aligned}
$$

where $\Lambda_{1}$ and $\Lambda_{2}$ are two positive definite matrices, one may write:

$$
\begin{aligned}
H \dot{\sigma}+B \sigma+K_{D} \sigma & =H(\ddot{q}-\dddot{\zeta})+B(\ddot{q}-\ddot{\zeta})+\left(C^{\prime} \dot{q}+D\right)-\left(C^{\prime} \dot{q}+D\right)+K_{D} \sigma \\
& =\left(H \ddot{q}+B \ddot{q}+C^{\prime} \dot{q}+D\right)-\left(H \ddot{\zeta}+B \ddot{\zeta}+C^{\prime} \dot{q}+D\right)+K_{D} \sigma \\
& =v-\left(H \dddot{\zeta}+B \ddot{\zeta}+C^{\prime} \dot{q}+D\right)+K_{D} \sigma \\
& =-K_{D} \sigma+u_{0}+w^{\prime \prime}+K_{D} \sigma \\
& =u_{0}+w^{\prime \prime}
\end{aligned}
$$

where Eqn. (5) and the following equation hold:

$$
w^{\prime \prime}=H \ddot{\zeta}+B \ddot{\zeta}+C^{\prime} \dot{\zeta}+D
$$

Also, the controller vector of voltage commands is assumed to be:

$$
v=u_{0}-K_{D} \sigma
$$

which is a simple and easy to compute controller and similar to (18). Because of properties 1, 2, 3 and 5 , one may write:

$$
\left\|w^{\prime \prime}\right\| \leq \rho^{\prime \prime}
$$

where

$$
\begin{aligned}
& \rho^{\prime \prime}=\delta_{0}+\delta_{1}\|e\|+\delta_{2}\|e\|^{2}+\delta_{3}\|\dot{e}\|+\delta_{4}\|e\|\|\cdot\| \dot{e} \|=S^{\prime} \theta^{\prime} \\
& S^{\prime}=\left[\begin{array}{lllll}
1 & \|e\| & \|e\|^{2} & \|\dot{e}\| & \|e\| .\|\dot{e}\|
\end{array}\right] \\
& \theta^{\prime}=\left[\begin{array}{lllll}
\delta_{0} & \delta_{1} & \delta_{2} & \delta_{3} & \delta_{4}
\end{array}\right]^{T}
\end{aligned}
$$




\section{Stability Analysis of the Proposed Scheme}

By introducing the Lyapunov function as:

$V=1 / 2 \sigma^{T} H \sigma+1 / 2 \tilde{\theta}^{\prime} \gamma^{-1} \tilde{\theta}^{\prime}+K_{\varepsilon}^{-1} \varepsilon$

its time derivative will be:

$\dot{V}=1 / 2 \sigma^{T} \dot{M} \sigma+\sigma^{T} M \dot{\sigma}+\tilde{\theta}^{\prime T} \gamma^{-1} \dot{\tilde{\theta}}^{\prime}+K_{\varepsilon}^{-1} \dot{\varepsilon}$

Assuming

$\dot{\varepsilon}=-K_{\varepsilon} \varepsilon$

combining Eqns. (21), (27), (33) and (34) gives:

$\dot{V}=1 / 2 \sigma^{T} \dot{H} \sigma+\sigma^{T}\left(w^{\prime \prime}-B \sigma-K_{D} \sigma+u_{0}\right)+\tilde{\theta}^{T} \gamma^{-1}\left(-\gamma \delta^{T}\|\sigma\|\right)$

$+K_{\varepsilon}^{-1}\left(-K_{\varepsilon} \varepsilon\right)=1 / 2 \sigma^{T}(\dot{H}-2 B) \sigma+\sigma^{T}\left(w^{\prime \prime}-K_{D} \sigma+u_{0}\right)$

$+\tilde{\theta}^{T} \gamma^{-1}\left(-\gamma \delta^{\prime T}\|\sigma\|\right)+\varepsilon$

Using Eqn. (5) in Eqn. (35) yields;

$\dot{V}=1 / 2 \sigma^{T}\left(L^{\prime} \dot{M}-2 B\right) \sigma+\sigma^{T} w^{\prime \prime}-\sigma^{T} K_{D} \sigma+\sigma^{T} u_{0}-\tilde{\theta}^{T} S^{T}|\sigma|-\varepsilon$

$=1 / 2 \sigma^{T}\left(L^{\prime} \dot{M}-2 L^{\prime} \dot{M}-2 L^{\prime} C-2 R^{\prime} M\right) \sigma+\sigma^{T} w^{\prime \prime}-\sigma^{T} K_{D} \sigma+\sigma^{T} u_{0}-\tilde{\theta}^{T} S^{T}\left|\sigma^{T}\right| \sigma \|-\varepsilon$

$=1 / 2 \sigma^{T}\left(-L^{\prime} \dot{M}-2 L^{\prime} C-2 R^{\prime} M+2 L^{\prime} C-2 L^{\prime} C\right) \sigma+\sigma^{T} w^{\prime \prime}-\sigma^{T} K_{D} \sigma+\sigma^{T} u_{0}-\tilde{\theta}^{T} S^{\prime T} \mid\|\sigma\|-\sigma$

$=-1 / 2 \sigma^{T} L^{\prime}(\dot{M}-2 C) \sigma-\sigma^{T}\left(2 L^{\prime} C+R^{\prime} M+K_{D}\right) \sigma+\sigma^{T} w^{\prime \prime}+\sigma^{T} u_{0}-\tilde{\theta}^{T} S^{T}\|\sigma\|-\varepsilon$

Since $L^{\prime}=l^{\prime} . I_{n \times n}$, by property $4, L^{\prime}(\dot{M}-2 C)$ is still a skew-symmetric matrix and one may conclude that:

$\dot{V}=-\sigma^{T}\left(2 L^{\prime} C+R^{\prime} M+K_{D}\right) \sigma \sigma^{T} w^{\prime \prime}+\sigma^{T} u_{0}-\tilde{\theta}^{\prime T} S^{\prime T}\|\sigma\|-\varepsilon$

Eqns. (14), (15), (17), (22) and (37) give:

$\dot{V} \leq \sigma^{T} \rho^{\prime \prime}+\sigma^{T} u_{0}-\sigma^{T}\left(2 L^{\prime} C+R^{\prime} M+K_{D}\right) \sigma-\tilde{\theta}^{T} S^{\prime T}\|\sigma\|-\varepsilon$

$=\sigma^{T} S^{\prime} \theta+\sigma^{T} u_{0}-\sigma^{T}\left(2 L^{\prime} C+R^{\prime} M+K_{D}\right) \sigma-\tilde{\theta}^{T} S^{T}\|\sigma\|-\varepsilon$

$\leq \theta^{\prime T} S^{\prime T}\|\sigma\|+\sigma^{T} u_{0}-\sigma^{T}\left(2 L^{\prime} C+R^{\prime} M+K_{D}\right) \sigma-\tilde{\theta}^{\prime T} S^{\prime T}\|\sigma\|-\varepsilon$

$=\hat{\theta}^{T} S^{T}\|\sigma\|+\sigma^{T} u_{0}-\sigma^{T}\left(2 L^{\prime} C+R^{\prime} M+K_{D}\right) \sigma-\varepsilon$

$\leq \hat{\theta}^{T} S^{T}\|\sigma\|-\sigma^{T} \sigma\left(\hat{\rho}^{2} /(\varepsilon+\hat{\rho} \| \sigma \mid)\right)-\sigma^{T}\left(2 L^{\prime} C+R^{\prime} M+K_{D}\right) \sigma-\varepsilon$

$=\hat{\theta}^{T} S^{\prime T}\|\sigma\|-\sigma^{T} \sigma\left(\left(S^{\prime} \hat{\theta}^{\prime}\right)^{2} /\left(\varepsilon+S^{\prime} \hat{\theta}\|\sigma\|\right)-\sigma^{T}\left(2 L^{\prime} C+R^{\prime} M+K_{D}\right) \sigma-\varepsilon\right.$

$=\hat{\theta}^{T} S^{T}\|\sigma\|-\|\sigma\|^{2}\left(\left(S^{\prime} \hat{\theta}^{\prime}\right)^{2} /\left(\varepsilon+S^{\prime} \hat{\theta}\|\sigma\|\right)\right)-\sigma^{T}\left(2 L^{\prime} C+R^{\prime} M+K_{D}\right) \sigma-\varepsilon$

$=\frac{\left(S^{\prime} \hat{\theta}^{\prime}\right)^{2}\|\sigma\|^{2}+\varepsilon S^{\prime} \hat{\theta}^{\prime}\|\sigma\|-\left(S^{\prime} \hat{\theta}^{\prime}\right)^{2}\|\sigma\|^{2}}{\varepsilon+S^{\prime} \hat{\theta}^{\prime}\|\sigma\|}-\sigma^{T}\left(2 L^{\prime} C+R^{\prime} M+K_{D}\right) \sigma-\varepsilon$

$=\frac{\varepsilon S^{\prime} \hat{\theta}^{\prime} \mid \sigma \|}{\varepsilon+S^{\prime} \hat{\theta}^{\prime}\|\sigma\|}-\varepsilon-\sigma^{T}\left(2 L^{\prime} C+R^{\prime} M+K_{D}\right) \sigma$

$=\frac{\varepsilon S^{\prime} \hat{\theta}^{\prime \prime}\|\sigma\|-\varepsilon^{2}-\varepsilon S^{\prime} \hat{\theta}^{\prime}\|\sigma\|}{\varepsilon+S^{\prime} \hat{\theta}^{\prime}\|\sigma\|}-\sigma^{T}\left(2 L^{\prime} C+R^{\prime} M+K_{D}\right) \sigma$

$=-\frac{\varepsilon^{2}}{\varepsilon+S^{\prime} \hat{\theta}^{\prime}\|\sigma\|}-\sigma^{T}\left(2 L^{\prime} C+R^{\prime} M+K_{D}\right) \sigma$

$\leq-\sigma^{T}\left(2 L^{\prime} C+R^{\prime} M+K_{D}\right) \sigma$

Considering that $K_{D}$ elements are usually chosen through a trial and error procedure and by taking advantage of properties 1 and 2, presence of the term $2 L^{\prime} C+R^{\prime} M$ is not a serious problem for the algorithm. Also, if the diagonal elements of $L^{\prime}$ are not equal, there still can be found a smaller region for $K_{D}$ elements that renders a stable closed loop system. Therefore, one may write:

$\dot{V} \leq-\lambda_{\min }\left(2 L^{\prime} C+R^{\prime} M+K_{D}\right)\|\sigma\|^{2}$

where $\lambda_{\min }$ is the smallest eigenvalue of matrix $2 L^{\prime} C+R^{\prime} M+K_{D}$. This implies that:

$V(0)-V(\infty) \geq \lambda_{\min } \int_{0}^{\infty}\|\sigma(t)\| d t$

Since $\dot{V}$ is negative semi-definite, it can be stated that $V$ is a non-increasing function and, therefore, it is upper bounded by $V(0)$, then:

$\lambda_{\min } \int_{0}^{\infty}\|r(\sigma)\| d \sigma<\infty$

or: 


$$
\sqrt{\int_{0}^{\infty}\|\sigma(t)\|^{2} d t}<\infty
$$

which shows $\sigma \in L_{2}^{n}$. To establish a stability result for the position tracking error, $\tilde{q}$, one may write the transfer function relationship between the tracking error and the filtered tracking error, $\sigma$, as:

$$
\tilde{q}(s)=G(s) \cdot \sigma(s)=\left(s^{2} I+s \Lambda_{1}+\Lambda_{2}\right)^{-1} \cdot \sigma(s)
$$

where $s$ is the Laplace variable. Since $G(s)$ is a strictly proper, asymptotically stable transfer function and $\sigma \in L_{2}^{\infty}$, one may conclude that, $[11,16]$ :

$$
\lim _{t \rightarrow \infty} \tilde{\mathrm{q}}=0
$$

Therefore, the position tracking error, $\tilde{q}$, and also the velocity tracking error, $\dot{\tilde{q}}$, are asymptotically stable.

\section{The Robotic System and the Desired Trajectory}

The considered robotic system used in this paper as the example to study the proposed control schemes is a 5-DOF RLED robot manipulator with revolute joints and DC motors as their actuators. A schematic of the robot is shown in, Fig. (1). Robot's kinematic and dynamic parameters and its motors' specifications can be found in Table (1) and Table (2) and [18].

Desired Trajectory is presented in joint space. It is designed so that in its first part, all joints have their accelerated motions together, then they move with constant velocities and in the final part, they will have separate decelerated motions, (See Fig. (2)). Therefore, in this trajectory all kinds of motion have been included.

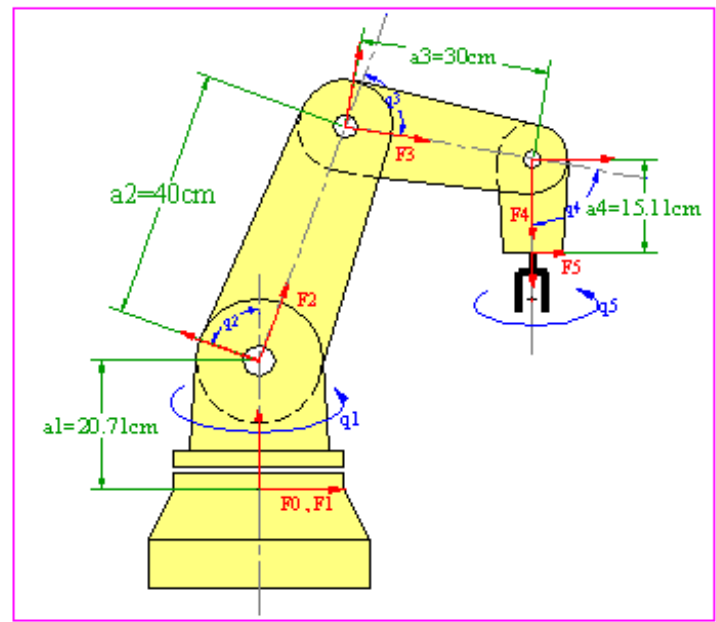

Figure 1. A schematic of the 5 DOF robot

\section{Simulation Results and Discussion}

The adaptive robust algorithm, described in the previous sections, has been applied on a 5 DOF RLED robot manipulator. The simulations are performed for two cases: 1) without any disturbances and 2) with sinusoidal disturbance on the control voltage commands as: $v_{d}=\left[\begin{array}{lllll}0.1 & 0.1 & 0.1 & 0.1 & 0.1\end{array}\right]^{T} \cdot \sin (\pi . t / 4)$. Simulation results of the control voltage commands and errors in joint position tracking are shown in Figs. 3-6.

Table 1. Kinematic (Denavit-Hartenberg) and Dynamic Parameters of the Robot

\begin{tabular}{|l|l|l|l|l|l|l|l|}
\hline Link & $\begin{array}{l}\text { Link } \\
\text { Variable }\end{array}$ & $\alpha_{i}$ [degree] & $\begin{array}{l}a_{i} \\
{[\mathrm{~mm}]}\end{array}$ & $\begin{array}{l}d_{i} \\
{[\mathrm{~mm}]}\end{array}$ & $\begin{array}{l}\text { Region of } \theta_{i} \\
{[\text { degree] }}\end{array}$ & Mass of Link [Kg] & $\begin{array}{l}\text { Position of } \mathbf{C}_{\mathrm{G}} \\
{[\mathrm{m}]}\end{array}$ \\
\hline $\mathbf{0}$ & - & - & - & 130 & - & - & \\
\hline $\mathbf{1}$ & $\theta_{1}$ & -90 & 0 & 125 & \pm 100 & 0.800 & 0.09 \\
\hline $\mathbf{2}$ & $\theta_{2}$ & 0 & 200 & 0 & 160 to 360 & 0.600 & 0.09 \\
\hline $\mathbf{3}$ & $\theta_{3}$ & 0 & 200 & 0 & \pm 100 & 0.400 & 0.09 \\
\hline $\mathbf{4}$ & $\theta_{4}$ & -90 & 0 & 0 & \pm 100 & 0.200 & 0.01 \\
\hline $\mathbf{5}$ & $\theta_{5}$ & 0 & 0 & 148 & \pm 200 & 0.100 & 0.06 \\
\hline
\end{tabular}




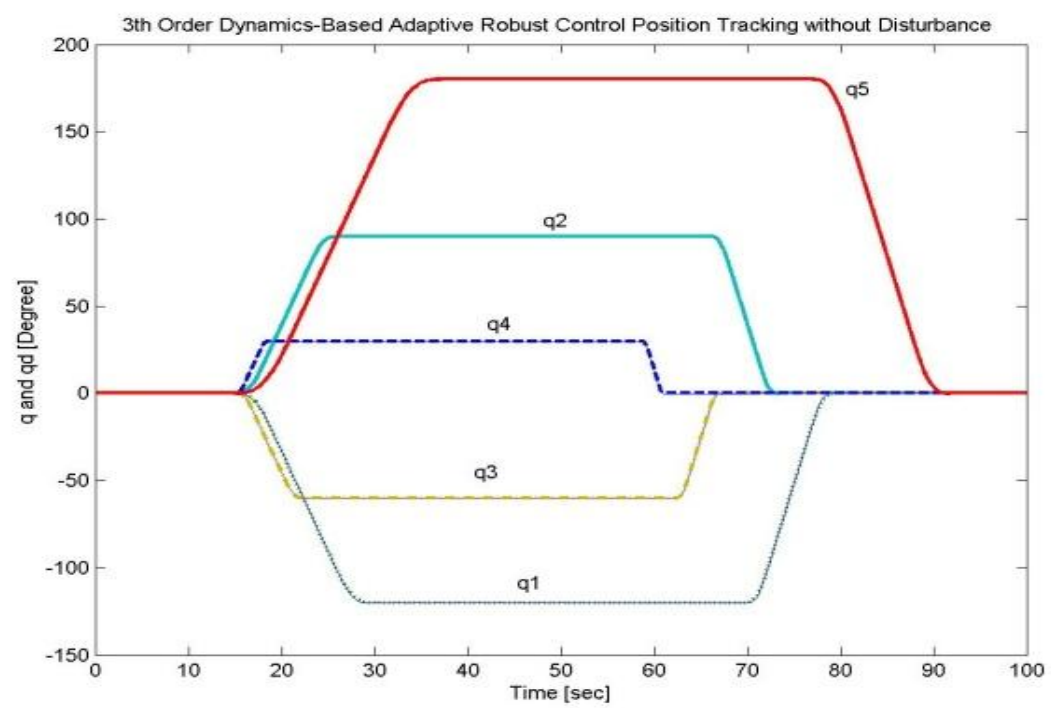

Figure 2. The desired trajectory

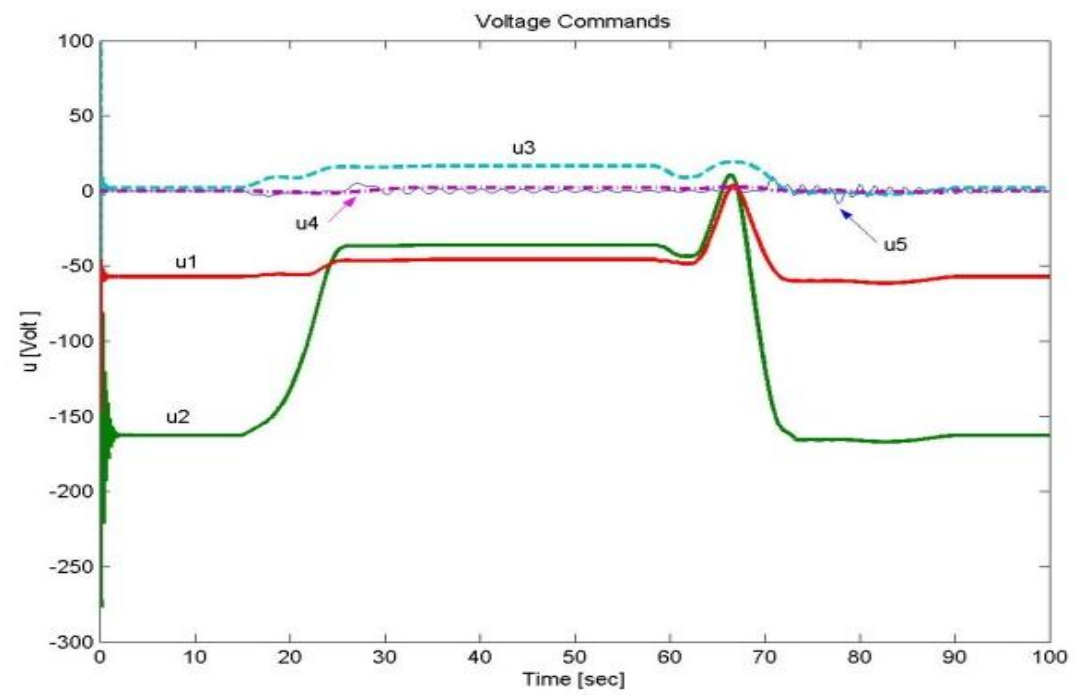

Figure 3. Voltage control commands by adaptive robust without any disturbances

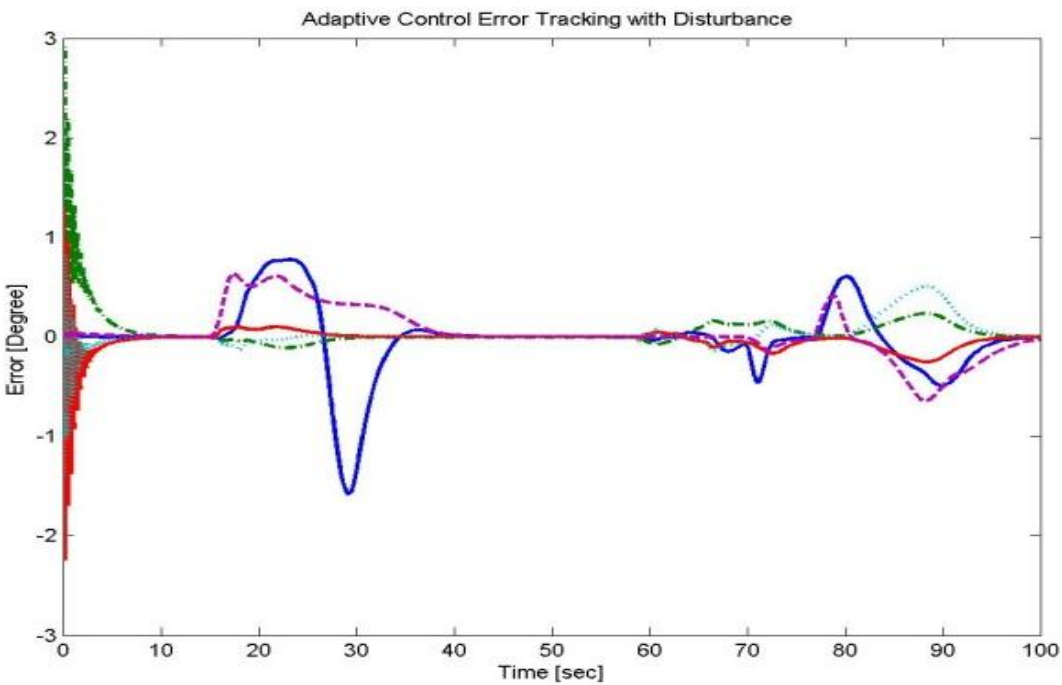

Figure 4. Errors in position tracking by adaptive robust control without any disturbances 


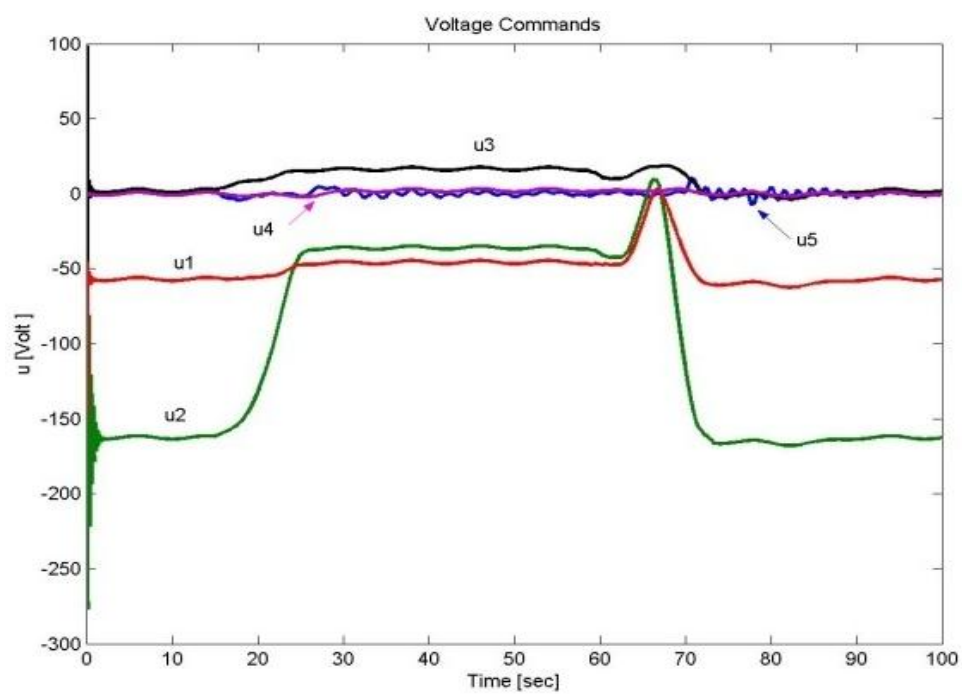

Figure 5. Control voltage commands by adaptive robust with sinusoidal disturbance



Figure 6. Errors in position tracking by adaptive robust control with sinusoidal disturbance

\section{Conclusion}

A In this paper, a combined third order dynamics for general non-redundant RLED robot manipulators' arm and actuator dynamics was adopted and an adaptive robust control method was presented for its trajectory tracking. Stability of the proposed scheme was proved using the Lyapunov theory. Simulation results of application of this method on a 5 DOF RLED robot show its good tracking performance and excellent robustness to disturbances. But the method needs joint acceleration measurement. Adaptive robust control method with third order dynamics, has a very good advantage of its very low computational burden (8 order less than that of adaptive control $[18,19]$ because of its very simple controlled voltage command and not requiring computation of the complex regressor matrix of the adaptive controllers. Comparison of the results of simulation of this scheme with those of two other adaptive(/robust) tracking control methods for RLED robots illustrates the effectiveness of the proposed scheme and demonstrates that this method is better in almost every respect but that it needs joint acceleration feedback [19].

\section{Appendix}

Time derivative of the Lyapunov function given in Eqn. (23) can be taken as:

$$
\dot{V}=1 / 2 \sigma^{T} \dot{M} \sigma+\sigma^{T} M \dot{\sigma}+\tilde{\theta}^{T} \gamma^{-1} \dot{\tilde{\theta}}+K_{\varepsilon}^{-1} \dot{\varepsilon}
$$

Considering Eqns. (10), (21), (34) and (46): 


$$
\begin{aligned}
& \dot{V}=1 / 2 \sigma^{T} \dot{M} \sigma+\sigma^{T}\left(w^{\prime}-C \sigma-K_{D} \sigma+u_{0}\right) \\
& +\tilde{\theta}^{T} \gamma^{-1}\left(-\gamma S^{T}\|\sigma\|\right)+K_{\varepsilon}{ }^{-1}\left(-K_{\varepsilon} \varepsilon\right) \\
& =1 / 2 \sigma^{T}(\dot{M}-2 C) \sigma+\sigma^{T} w^{\prime}+\sigma^{T} u_{0} \\
& -\sigma^{T} K_{D} \sigma-\tilde{\theta}^{T} S^{T}\|\sigma\|-\varepsilon
\end{aligned}
$$

Because of property 4 and taking into account Eqns. (14), (15), (17) and (22), one may obtain:

$$
\begin{aligned}
& \dot{V}=\sigma^{T} w^{\prime}+\sigma^{T} u_{0}-\sigma^{T} K_{D} \sigma-\tilde{\theta}^{T} S^{T}\|\sigma\|-\varepsilon \\
& \leq \sigma^{T} \rho+\sigma^{T} u_{0}-\sigma^{T} K_{D} \sigma-\tilde{\theta}^{T} S^{T}\|\sigma\|-\varepsilon \\
& =\sigma^{T} S \theta+\sigma^{T} u_{0}-\sigma^{T} K_{D} \sigma-\tilde{\theta}^{T} S^{T}\|\sigma\|-\varepsilon \\
& \leq \theta^{T} S^{T}\|\sigma\|+\sigma^{T} u_{0}-\sigma^{T} K_{D} \sigma-\tilde{\theta}^{T} S^{T}\|\sigma\|-\varepsilon \\
& =\hat{\theta}^{T} S^{T}\|\sigma\|+\sigma^{T} u_{0}-\sigma^{T} K_{D} \sigma-\varepsilon \\
& \leq \hat{\theta}^{T} S^{T}\|\sigma\|-\sigma^{T} \sigma \frac{\hat{\rho}^{2}}{\varepsilon+\hat{\rho}\|\sigma\|}-\sigma^{T} K_{D} \sigma-\varepsilon \\
& =\hat{\theta}^{T} S^{T}\|\sigma\|-\sigma^{T} \sigma \frac{(S \hat{\theta})^{2}}{\varepsilon+S \hat{\theta}\|\sigma\|}-\sigma^{T} K_{D} \sigma-\varepsilon \\
& =\hat{\theta}^{T} S^{T}\|\sigma\|-\|\sigma\|^{2} \frac{(S \hat{\theta})^{2}}{\varepsilon+S \hat{\theta}\|\sigma\|}-\sigma^{T} K_{D} \sigma-\varepsilon \\
& =\frac{(S \hat{\theta})^{2}\|\sigma\|^{2}+\varepsilon S \hat{\theta}\|\sigma\|-(S \hat{\theta})^{2}\|\sigma\|^{2}}{\varepsilon+S \hat{\theta}\|\sigma\|}-\sigma^{T} K_{D} \sigma-\varepsilon \\
& =\frac{\varepsilon S \hat{\theta}\|\sigma\|}{\varepsilon+S \hat{\theta}\|\sigma\|}-\varepsilon-\sigma^{T} K_{D} \sigma \\
& =\frac{-\varepsilon S \hat{\theta}\|\sigma\|-\varepsilon^{2}+\varepsilon S \hat{\theta}\|\sigma\|}{\varepsilon+S \hat{\theta}\|\sigma\|}-\sigma^{T} K_{D} \sigma \\
& =-\frac{\varepsilon^{2}}{\varepsilon+S \hat{\theta}\|\sigma\|}-\sigma^{T} K_{D} \sigma \\
& \leq-\sigma^{T} K_{D} \sigma
\end{aligned}
$$

\section{References}

[1] T. Burg, D. M. Dawson, J. Hu and M. S. de Queiroz, An adaptive partial sate feedback controller for RLED robot manipulators, IEEE Transactions on Automatic Control, 41, 1996, 1024-1030.

[2] H. Canbolat, J. Hu, and D. M. Dawson, A hybrid learning/adaptive partial sate feedback controller for RLED robot manipulators, Proceedings of IEEE International Conference on Decision and Control, 1995, 39-44.

[3] C. Y. Su, and Y. Stepanenko, Backstepping based hybrid adaptive control of robot manipulators incorporating actuator dynamics, International Journal of Adaptive Control and Signal Processing, 1997, 141-153.

[4] C. Y. Su, and Y. Stepanenko, Redesign of hybrid adaptive/robust motion control of rigid-link electrically driven robot manipulators, Proceeding of IEEE Conference on Decision and Control, San Diego, CA, December 1997, 724-731.

[5] S. Tzafestas, G. Starrakakis, and L. Dritsas, Performance Analysis of the Computed Torque Method for Robotic Manipulators with Fast Actuators, IEEE International Conference on Control and Applications ICCON '89, 1989, 734 - 739.

[6] J. Yuan, Adaptive control of robotic manipulators including motor dynamics, IEEE Transactions on Robotics and Automation, 11, $1995,612-617$

[7] D. N. Kouya, M. Saad,,L. Lamarche, and C. Khairallah, Backstepping adaptive position control for robot manipulators, Proceeding of American Control Conference, 2001,636-640.

[8] D. N. Kouya, M. Saad, and,L. Lamarche, Backstepping passivity adaptive position control for robot manipulators, Proceeding of American Control Conference, 2002, 4607-4611.

[9] D. M. Dawson, Z. Qu, and J. J. Carrol, Tracking control of rigid link electrically driven robot manipulator, International Journal of Control, 1992, 911-1006.

[10] M. C. Good,L. M. Sweet, and K. L. Strobel, Dynamics models for control system design of integrated robot and drive systems, Journal of Dynamical Systems: Measurements and Control, 1985, 53-59.

[11] F. L. Lewis,C. T. Abdallah, and. M. Dawson, Control of Robot Manipulators, $2^{\text {nd }}$ edition (Macmillan Publishing Company, 2004).

[12] S. S. Ge, and I. Postlethwaite, Nonlinear Adaptive control of robots including motor dynamic, Proceeding of American Control Conference, San Francisco, CA, , 1993, 1423-1427,

[13] M. S.de Queiroz, D. M. Dawson, and H. Canbolat, Adaptive position/force control of BDC-RLED robots without velocity measurements, Proceedings of IEEE International Conference on Robotics and Automation, 1997, 525-530. 
[14] J. Guldner, J. J. Carroll, D. M. Dawson, and Z. Qu, Robust tracking control of rigid-link electrically-driven robots, Proceedings of IEEE International Conference on Decision and Control, 1992, 1866-1868.

[15] Y. Stepaneko,and C. Y. Su, Adaptive motion control of rigid-link electrically-driven robot manipulators, Proceedings of IEEE International Conference on Robotics and Automation, 1994, 630-635.

[16] C. Canudas De Wit, B. Siciliano, and G. Bastin, Theory of Robot Control, (Springer-Verlag, 1996).

[17] L. Sciavicco, and B. Siciliano, Modelling and Control of Robot Manipulators, Second Edition, (Springer, 2000).

[18] M. Mohseni, Adaptive Control of a 5 DOF Robot Manipulator Including Motor Dynamics, MSc. Thesis, School of Mechanical Engineering, Shiraz University, 2003

[19] M. Mohseni, M. Eghtesad, and A. Lotfazar, Adaptive control methods for trajectory tracking of a 5 DOF RLED robot manipulator: A Comparison, Proceedings of World Automation Congress, Sevilla, Sapin,2004, 179-184. 